\title{
Les statines, l'étendue des indications et les risques pour les enfants et les adolescents
}

$\mathrm{P}$ lus de la moitié des médicaments administrés aux enfants et aux jeunes à l'hôpital sont utilisés de façons non indiquées. Même si les traitements administrés aux adultes sont habituellement guidés par les données probantes, les données sur l'utilisation de ces médicaments chez les enfants sont souvent rares ou inexistantes. Les médecins sont donc confrontés à un dilemme : traiter sans données probantes et risquer des résultats indésirables graves ou refuser d'utiliser des médicaments qui peuvent sauver une vie ou en améliorer la qualité.

Le problème persiste même si l'on réclame depuis des années plus d'études sur l'innocuité et l'efficacité qui portent aussi sur les enfants. Les exemples de décès d'un enfant parce qu'un médicament n'a pas produit le même effet que chez l'adulte sont nombreux. Les enfants ne sont pas des adultes de petite taille, particulièrement en ce qui concerne les effets des médicaments. La pharmacocinétique, la pharmacodynamique, l'efficacité et les résultats indésirables peuvent différer considérablement.

Des recommandations récentes du Comité de la nutrition de l'American Academy of Pediatrics nous rappellent que le problème de l'étendue des indications aux enfants ne se limite pas aux médicaments utilisés à l'hôpital'. L'American Academy of Pediatrics recommande que l'on envisage une pharmacothérapie contre les dyslipidémies chez les patients d'à peine huit ans qui répondent à certains critères précis, y compris l'hypercholestérolémie familiale et non familiale ${ }^{1}$. Les statines sont au nombre des médicaments recommandés. La Food and Drug Administration des États-Unis a approuvé l'utilisation des statines chez les enfants pour traiter l'hypercholestérolémie familiale, mais pas pour traiter d'autres dyslipidémies.

Les preuves directes de l'innocuité et de l'efficacité des statines chez les enfants et les jeunes sont limitées. L'approbation de la Food and Drug Administration reposait sur des données extrapolées d'études portant sur des adultes, ainsi que sur des études de courte durée portant sur l'utilisation des statines chez les enfants et les adolescents atteints d'hypercholestérolémie familiale. À la suite de 11 études portant sur des enfants (qui avaient à peu près tous une hypercholestérolémie familiale) soit, au total, 665 patients recevant des statines et 331 témoins, on a signalé une diminution moyenne de $32 \%$ (intervalle de $17 \%$ à $45 \%$ ) des lipoprotéines de faible densité . Une seule étude a suivi les patients pendant plus d'un an. Aucune étude n'a évalué le bienfait clinique à long terme, par exemple une diminution du nombre des infarctus du myocarde des décennies plus tard. Les lignes directrices sur l'utilisation à long terme des statines chez les enfants et les jeunes ne reposent donc sur aucune preuve de leur efficacité ou de leur innocuité.

Nous commençons à peine à comprendre chez les adultes comment le risque de résultats indésirables graves, comme la rhabdomyolyse, qui découle de l'utilisation de statines est fonction de la variabilité de l'expression et de l'activité des systèmes de transport. On ne comprend pas encore chez l'enfant l'ontogénie et l'expression d'un grand nombre de ces systèmes clés, ce qui amplifie l'incertitude quant à l'innocuité à long terme. De plus, l'observation selon laquelle certaines sous-populations d'adultes peuvent présenter un risque élevé de toxicité reliée aux statines soulève d'autres préoccupations au sujet de leur toxicité éventuelle pour les enfants. En outre, certaines statines, y compris la simvastatine et la lovastatine, peuvent traverser la barrière hémato-encéphalique, ce qui est inquiétant parce que les lipides ont un rôle clé à jouer dans le développement normal du cerveau, sans oublier que le neurodéveloppement et l'intégration du traitement des signaux sont intenses au cours de l'adolescence et au début de l'âge adulte ${ }^{3}$. L'extrapolation de données sur l'innocuité dérivées des adultes, chez lesquels le développement du cerveau est terminé depuis longtemps, ou d'essais de courte durée portant sur des adolescents, ne suffit pas pour dissiper ces préoccupations.

En prescrivant des statines aux enfants et aux jeunes, on risque de les engager dans des décennies de traitement. Aux États-Unis, des incitatifs (p. ex., protection des brevets pendant plus longtemps) poussent les sociétés pharmaceutiques à réaliser, sur des enfants et des jeunes, des essais randomisés de nouveaux médicaments visant principalement un marché adulte mais que l'on pourrait utiliser chez les enfants. Il n'y a malheureusement pas d'incitatif à effectuer, après l'homologation, les études de cohorte de longue durée nécessaires pour déterminer l'efficacité et l'innocuité de traitements de longue durée. Sans ces études, nous risquons toutefois de répéter le fiasco de l'utilisation clinique fondée sur «de bonnes données scientifiques extrapolées» tirées de données à court terme, comme on l'a fait avec le diéthylstilbestrol.

Les organismes de réglementation des médicaments doivent pouvoir imposer des programmes de surveillance et d'évaluation actives chez les enfants et les jeunes qui prennent pendant longtemps un médicament comme les statines. Un financement insuffisant dans ce domaine expose nos enfants et nos jeunes à des séquelles inconnues. L'optimisation des résultats pour la santé dans ce groupe d'âge pourrait toutefois produire d'énormes retombées sur la santé publique étant donné leur trajectoire de vie. Entre-temps, les médecins qui traitent des enfants atteints de dyslipidémie sans hypercholestérolémie familiale doivent viser d'abord à assurer que l'on a envisagé complètement et à plusieurs reprises les solutions de rechange à la pharmacothérapie, comme l'alimentation, l'exercice et autres facteurs liés aux habitudes de vie. La facilité séduisante avec laquelle on peut prescrire une pilule peut constituer une décision mal avisée qui engage nos patients les plus jeunes, pour une période indéfinie, dans un parcours où les avantages et les préjudices sont inconnus.

Noni MacDonald MD MSc

Rédactrice de section, Santé publique

Matthew B. Stanbrook MD PhD

Rédacteur adjoint, Sciences, JAMC

Michael J. Rieder MD PhD

Chaire IRSC-GSK en pharmacologie clinique pédiatrique

Université Western Ontario, London (Ont.)

Avec l'équipe de rédaction de l'éditorial

(Paul C. Hébert MD MHSc, Barbara Sibbald BJ,

Ken Flegel MDCM MSc et Amir Attaran LLB DPhil).

Intérêts concurrents: Voir www.cmaj.ca/misc/edboard.shtml pour les déclarations de l'équipe de rédaction de l'éditorial. Aucuns déclarés pour Michael J. Rieder.

Traduit par le Service de traduction de l'AMC.

\section{RÉFÉRENCES}

1. Daniels SR, Greer FR, Committee on Nutrition. Lipid screening and cardiovascular health in childhood. Pediatrics 2008;122:198-208.

2. Tapia Ceballos L, Picazo Angelina B, Ruiz Garcia C. Uso de estatinas durante la infancia. An Pediatr (Barc) 2008;68:385-92.

3. Lebel C, Walker L, Leemans A, et al. Microstructural maturation of the human brain from childhood to adulthood. Neuroimage 2008;40:1044-55. 